\title{
Time-Resolved Optical Waveguide Spectroscopy for Studying Protein Adsorption Kinetics
}

\author{
Jose H. Santos ${ }^{1}$, Naoki Matsuda ${ }^{1}{ }^{*}$, Zhi-Mei Qi ${ }^{1}$, Takamitsu Yoshida ${ }^{1}$, \\ Akiko Takatsu ${ }^{2}$ and Kenji Kato ${ }^{2}$ \\ ${ }^{1}$ Nanoarchitectonics Research Center, AIST Central 5, Tsukuba 305-8565, Japan \\ ${ }^{2}$ National Metrology Institute of Japan, AIST Central 3, Tsukuba 305-8563, Japan
}

\begin{abstract}
A time-resolved evanescent wave absorption technique is employed in studying the adsorption of a mammalian protein (cytochrome $c$ ) from an electrolyte-free solution on a hydrophilic glass surface. The method combines the sensitivity of a fast-scan spectral analyzer and surface specificity of slab optical waveguide (SOWG) technique. While proteins are viewed to undergo an overall irreversible adsorption process brought about by structural changes that occur after the initial adsorption phase, we have used an SOWG technique to investigate the first stage of the adsorption process while no noticeable changes have taken place. Absorbance data within the first two seconds after contact fit well into the reversible adsorption model characteristic of Langmuir-type adsorption but the goodness-of-fit diminishes as the protein molecules spend more time on the surface.
\end{abstract}

(Received October 17, 2003; Accepted December 19, 2003)

Keywords: protein adsorption, evanescent wave, slab optical waveguide spectroscopy, cytochrome c, time-resolved spectroscopy

\section{Introduction}

Biocompatibility is an essential feature of any materials that are being developed and used for biomedical applications. ${ }^{1)}$ The demand for implants to replace damaged or diseased joints, teeth and other body structures underscores the urgent need for scientific investigations in this field. ${ }^{2)}$ One of the most important phenomena involved in biocompatibility is surface adsorption and the present paper is an effort to contribute in this area of research.

The propagation of electromagnetic radiation in a waveguide structure through internal total reflection (ITR) is wellknown and has been utilized in a number of analytical techniques for studying surface adsorption. ${ }^{3,4)}$ Some popular examples of these techniques are ellipsometry, total internal reflection fluorescence (TIRF) and surface plasmon resonance (SPR) methods. The surface sensitivity of ITR-based methods is mainly attributed to the formation of evanescent wave at each internal reflection within the waveguide. Depending on the wavelength of light and its angle of incidence, the depth of the evanescent field varies from around one-quarter of the wavelength used to a value less than that wavelength. ${ }^{4,5)}$

Slab optical waveguide (SOWG) absorption spectroscopy is a technique based on the sample absorption at the evanescent field in a planar internal reflection element. The waveguide is composed of a thin film, characterized by a relatively high refractive index, surrounded by two semiinfinite bounding media of somewhat lower refractive indices. For several years now, our group has been developing this technique and tried a number of ways to investigate interfacial phenomena involving proteins and dye molecules, particularly adsorption processes, by measuring the transmission and absorbance spectra of the reflected light using various waveguide materials. ${ }^{6-14)}$ Recently, we have developed a cell arrangement wherein a polychromatic light is

*Author to whom correspondence should be addressed transmitted within the waveguide using a glycerol drop in lieu of conventional prism or grating couplers. ${ }^{6,7)}$ We have combined this novel cell arrangement with the high-speed photonic charge-coupled device to observe the adsorption behavior of proteins right after solution-surface contact. In this paper, we reported a simple lab-built SOWG platform that allows absorbance measurement at the evanescent field in millisecond timescale for probing the kinetics of adsorption of cytochrome $c$ molecules at a hydrophilic glass surface.

The surface activities of proteins vary appreciably due to the differences in physicochemical properties that are influenced largely by external factors such as $\mathrm{pH}$, ionic strength and temperature. These factors change the molecular and conformational stability at interfaces and the nature and distribution of hydrophilic and hydrophobic residues and sites on both the adsorbing surface and protein molecules. The success of each collision with the interface leading to adsorption is a function of hydrophobicity-hydrophilicity ratio of the interacting surfaces. The present study performed on cytochrome $c$ does not represent the analysis of adsorption behavior of proteins onto hydrophilic surface, but rather an attempt to utilize the reported method in studying specific protein interactions with the hydrophilic sites arrayed on glass surface.

\section{Experimental}

Horse heart cytochrome $c$ (C-7752, 95\%) was obtained from Sigma and used as received. Protein solutions were prepared using doubly distilled deionized water and filtered using Millex GV syringe driven filter unit equipped with $0.22 \mu \mathrm{m}$ Durapore PVDF membrane (Sigma).

Absorbance measurements were performed using a SOWG system fabricated in our laboratory and shown schematically in Figs. 1(a) and (b). A Xe150W Lamp Unit (SIC, Japan) was used as the light source, while the detector was a Photonic Mutichannel Analyzer from Hamamatsu (Model C7473, Japan). The incident light is conveniently guided into the 

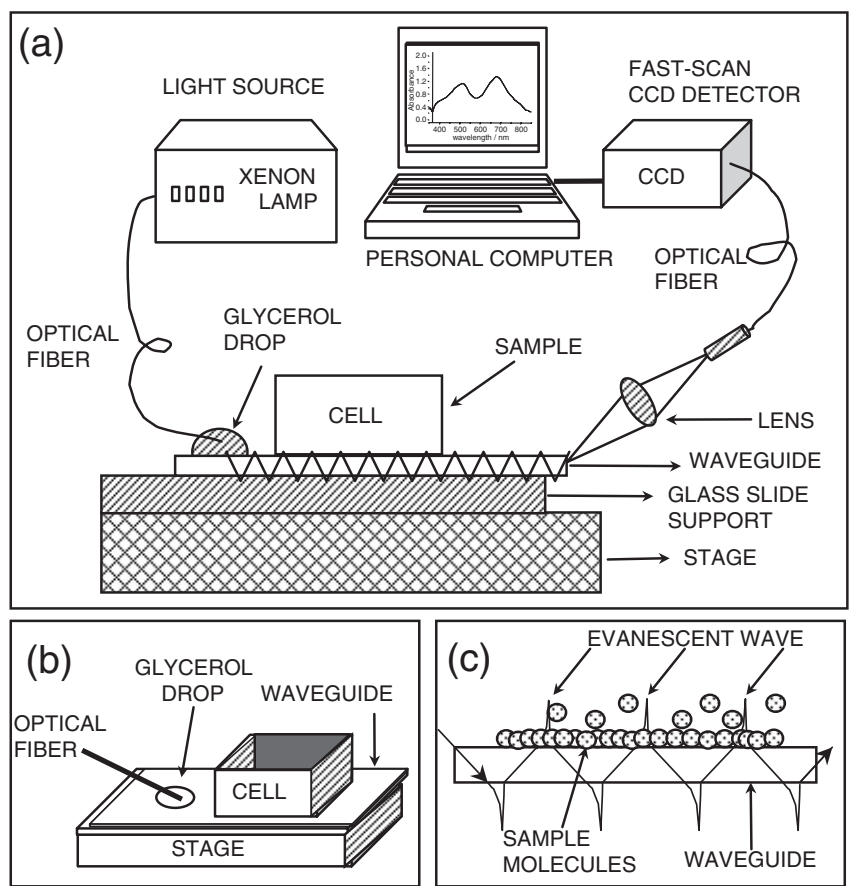

Fig. 1 Schematic diagram of the SOWG setup showing all the relevant components (a), a three-dimensional view of the cell (b), and the simplified illustration of evanescent wave absorption by the sample molecules on the surface (c).

waveguide by immersing the tip of an optical fiber from the light source in a droplet of glycerol laid on top of the glass surface. The guided light undergoes a series of multiple internal reflections before it is being transmitted and detected at the end of the waveguide. In Fig. 1(c), a schematic illustration of the absorption of the evanescent wave by the sample molecules at the surface is also shown. The SOWG cell is composed of a $50-\mu \mathrm{m}$ thin glass plate supported in an ordinary microscope slide. The glass slide is lined with a black electrical tape to minimize the influence of stray light and fitted with 1-mm slit at the center to provide an air medium with lower refractive index along the light path. This arrangement allows for maximum amount of reflected light being transmitted at the end of the waveguide into the objective lens system. Prior to each experiment, the glass surface to be used was equilibrated with pure water until the blank absorbance stayed at zero value. The sample was introduced through a $1.5 \times 1.0-\mathrm{cm}$ rectangular hole made of silicone rubber sheet mounted on top of the glass waveguide surface. Several absorbance measurements were performed on identical conditions to establish the reproducibility of the technique. The reported data were average of two or three sets of data that gave the most reproducible results. Nonlinear curve fitting analyses were performed using GraphPad Prism $^{\mathrm{TM}}$ software.

\section{Results and Discussion}

The adsorption of proteins on surfaces is a very important phenomenon in many biochemical applications due to recognized influence of surface processes on the activity of these biomolecules. In many cases, protein adsorption is the central event in the biofouling of surfaces involved in cell growth in cultures, ${ }^{15)}$ biomedical implants ${ }^{16)}$ as well as in biosensor systems. ${ }^{17,18)}$ In this regard, it is not only the amount of adsorption that is vital in these systems but the overall mechanism and kinetics by which the whole adsorption process occurs. It is therefore necessary to observe the surface processes from the shortest possible time and in the most identical environment for which these molecules function. Through that, we can better understand the details of physical and chemical interactions that these molecules undertake throughout the course of adsorption.

We have followed the adsorption of protein molecules on a hydrophilic glass surface by measuring the absorbance spectra of the reflected polychromatic light in millisecond timescale. Absorbance measurements were taken every $50 \mathrm{~ms}$ right after protein solution-surface contact for a period of $10 \mathrm{~s}$. Cytochrome $c$, a mammalian protein, was used and the collected data were compared with the simple and extended adsorption models typifying reversible and less reversible adsorption processes, respectively. $R$-squared $\left(R^{2}\right)$ goodness-of-fit tests were performed to evaluate the closeness of the observed data with these mathematical models. The absorption spectra of cytochrome $c$ right after the introduction of $5 \mu \mathrm{M}$ solution into the SOWG cell are shown in Fig. 2(a). The bottom curve was recorded $50 \mathrm{~ms}$ after contact and the succeeding curves on top were recorded every $50 \mathrm{~ms}$ within a total residence time of $10 \mathrm{~s}$. In Fig. 2(b), a summary of absorbance at $409 \mathrm{~nm}$, which corresponds to the wavelength of maximum absorption, is given for various bulk concentrations of cytochrome $c$. All the subsequent calculations presented here are based on the assumption that the measured absorbance is a direct measure of the amount of adsorbed molecules on the surface. ${ }^{7)}$
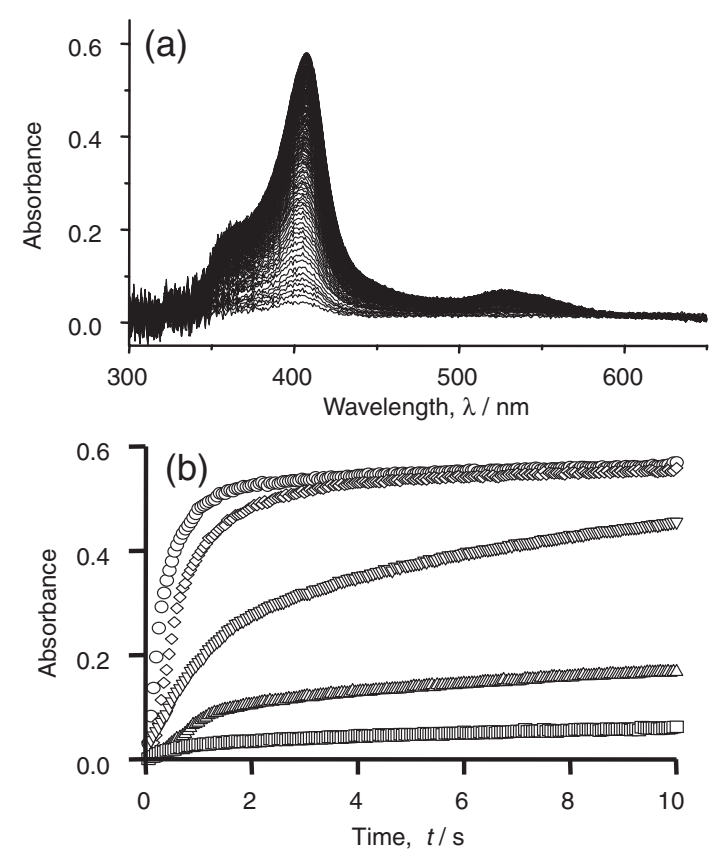

Fig. 2 SOWG absorbance data for kinetic studies of the adsorption of cytochrome $c$ on glass surface in the absence of electrolyte: (a) superimposed spectra collected every $50 \mathrm{~ms}$ right after sample introduction; (b) summary of absorbance at $409 \mathrm{~nm}$ for $(\square) 0.5,(\triangle) 1.0,(\nabla) 2.0$, $(\diamond) 5.0$, and $(\bigcirc) 10.0 \mu \mathrm{M}$ bulk concentrations. 


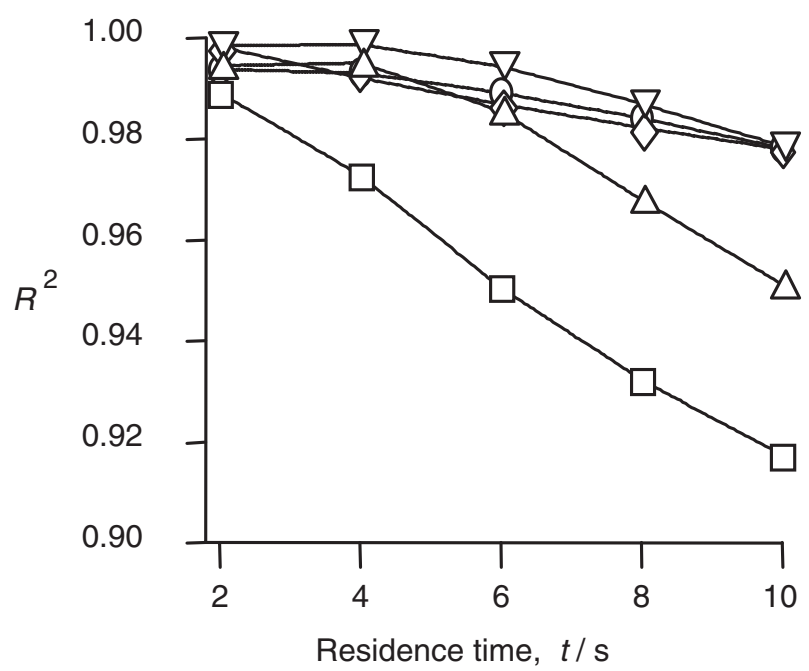

Fig. 3 Plots of curve-fitting $R^{2}$ goodness-of-fit test results derived from time-resolved SOWG absorption data for the kinetic studies of the adsorption of cytochrome $c$ on glass surface in the absence of electrolyte: $(\square) 0.5,(\triangle) 1.0,(\nabla) 2.0,(\diamond) 5.0$, and $(\bigcirc) 10.0 \mu \mathrm{M}$ bulk concentrations.

To determine the agreement of the experimental data with the generally accepted adsorption kinetic equation, we used a fitting algorithm using the simple adsorption model, ${ }^{19)}$

$$
A=A_{\max }[(1-\exp (-b t))]
$$

where $A$ is the measured absorbance, $A_{\max }$ is the steady-state absorbance, $b$ is the observed rate constant $\left(\mathrm{s}^{-1}\right)$ and $t$ is the time elapsed after surface-solution contact. The collected time-resolved data were fitted into the simple adsorption model using the absorbance values gathered after 2, 4, 6, 8 and $10 \mathrm{~s}$ residence times. The resulting $R^{2}$ goodness-of-fit test results computed from the sum of the squares of the distances of the points from the best fit curve are shown in Fig. 3. For all bulk concentrations used, the $R^{2}$ values are consistently very close to unity at $2 \mathrm{~s}$ residence time and decreases with increasing residence time especially with samples having lower cytochrome $c$ concentrations. This trend suggests that deviation of the observed data with the model becomes more significant with increasing residence time and one may infer that structural changes might be taking place as the protein molecules spend longer time on the surface. This observation is dramatically demonstrated in Figs. 4(a) and (b) where the same model was used for fitting the data obtained for 2 and $10 \mathrm{~s}$ residence times, respectively. The model curve fitted well with the data obtained within $2 \mathrm{~s}$ but not so well with those within $10 \mathrm{~s}$.

For the purpose of finding a model that suits better with the observed data at longer residence time, we have used the extended adsorption model,

$$
A=A_{1}\left[\left(1-\exp \left(-b_{1} t\right)\right)\right]+A_{2}\left[\left(1-\exp \left(-b_{2} t\right)\right)\right]
$$

where the additional term, $A_{2}\left[\left(1-\exp \left(-b_{2} t\right)\right)\right]$, covers the extension for irreversibility. The data gathered within $10 \mathrm{~s}$ are fitted into the extended adsorption model and the results are shown in Fig. 4(c). The calculated $R^{2}$ value for this fitting test was a higher 0.995 compared to 0.917 obtained when the simple adsorption model was used (Fig. 4(b)). Noting that the first term in the extended adsorption model is identical to the
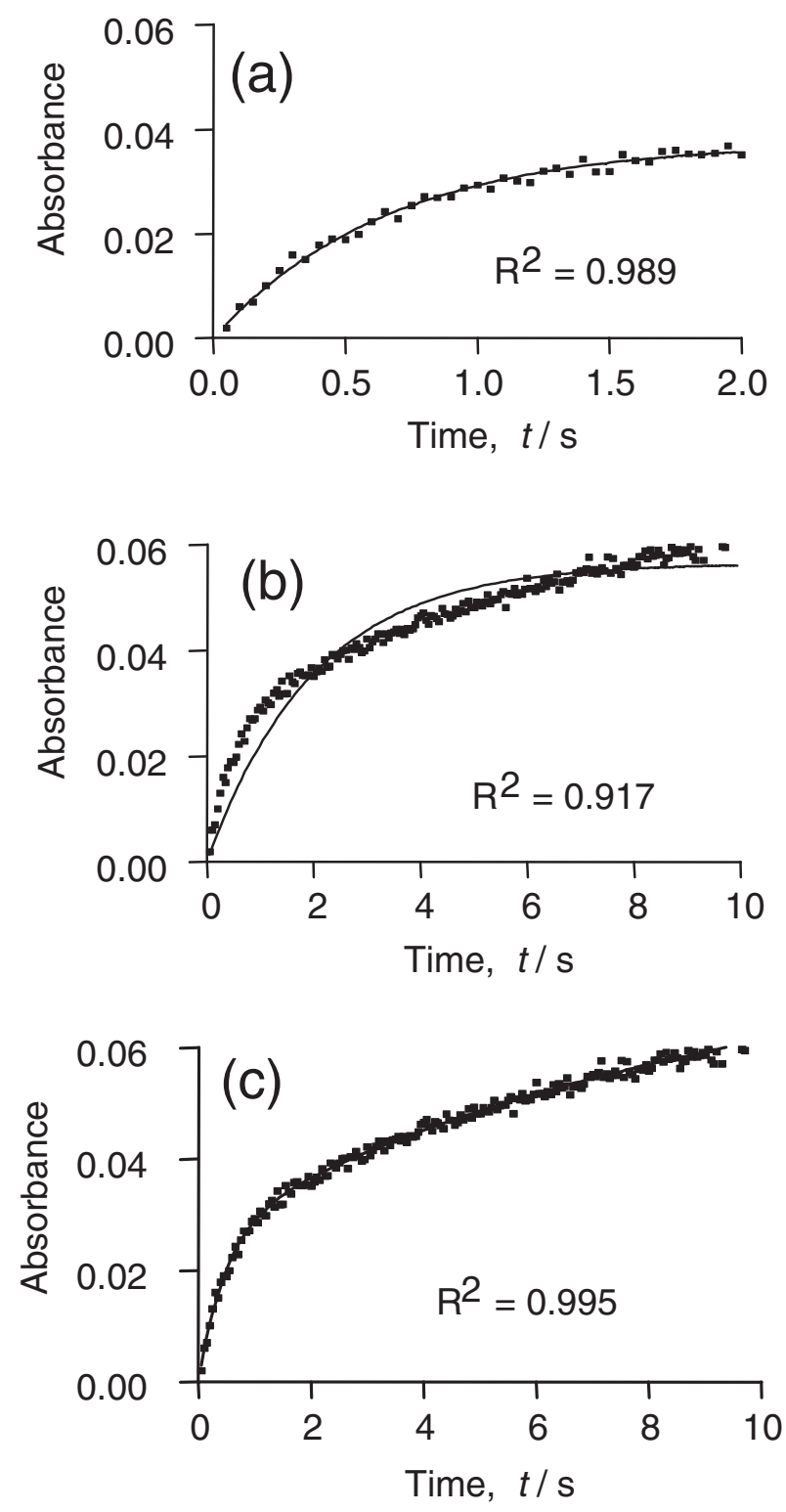

Fig. 4 SOWG absorbance data (dots) at $409 \mathrm{~nm}$ for $0.5 \mu \mathrm{M}$ cytochrome $c$ in water within $2 \mathrm{~s}$ (a) and $10 \mathrm{~s}$ (b) after solution-glass surface contact showing the curve-fitting results (continuous line) using the simple adsorption model. Plot (c) is the same experimental data in (b) fitted using the extended adsorption model (continuous line).

single term in the simple adsorption model, we propose that the second term is the contribution of some factors other than the Langmuir-type adsorption of protein molecules.

The parameters derived from curve-fitting of the data presented in Fig. 2(b) are summarized in Table 1. For all cytochrome $c$ solutions studied, the $R^{2}$ value decreases with increasing residence time. Consequently, the calculated maximum absorbance $\left(A_{\max }\right)$ also increases while the rate constant $(b)$ decreases as the residence time is increased. These results demonstrated that cytochrome $c$ molecules showed a strong dependence (based on $R^{2}$ values approaching unity) on the simple adsorption model within $2 \mathrm{~s}$ after contact and the dependence weaken upon increasing the residence time. Interestingly, at longer residence times, the observed data developed to fit well into the extended adsorption model that strongly suggested post adsorption 
Table 1 Summary of derived parameters obtained by fitting the experimental data into the simple adsorption model.

\begin{tabular}{|c|c|c|c|c|c|c|}
\hline \multirow{2}{*}{$\begin{array}{c}\text { [Cyt. c] } \\
(\mu \mathrm{M})\end{array}$} & \multirow{2}{*}{ Parameter } & \multicolumn{5}{|c|}{ Contact time, $t_{\mathrm{c}} / \mathrm{s}$} \\
\hline & & 2 & 4 & 6 & 8 & 10 \\
\hline \multirow{3}{*}{0.5} & $A_{\max }$ & 0.038 & 0.043 & 0.048 & 0.053 & 0.057 \\
\hline & $b$ & 1.5 & 1.1 & 0.79 & 0.62 & 0.50 \\
\hline & $R^{2}$ & 0.989 & 0.973 & 0.951 & 0.932 & 0.917 \\
\hline \multirow{3}{*}{1.0} & $A_{\max }$ & 0.13 & 0.13 & 0.14 & 0.15 & 0.16 \\
\hline & $b$ & 0.96 & 0.94 & 0.80 & 0.67 & 0.57 \\
\hline & $R^{2}$ & 0.995 & 0.995 & 0.986 & 0.968 & 0.951 \\
\hline \multirow{3}{*}{2.0} & $A_{\max }$ & 0.36 & 0.36 & 0.39 & 0.41 & 0.43 \\
\hline & $b$ & 0.70 & 0.70 & 0.61 & 0.53 & 0.46 \\
\hline & $R^{2}$ & 0.999 & 0.999 & 0.994 & 0.987 & 0.979 \\
\hline \multirow{2}{*}{5.0} & $A_{\max }$ & 0.49 & 0.52 & 0.53 & 0.54 & 0.54 \\
\hline & $R^{2}$ & 0.998 & 0.992 & 0.987 & 0.982 & 0.978 \\
\hline \multirow{3}{*}{10.0} & $A_{\max }$ & 0.53 & 0.53 & 0.54 & 0.55 & 0.55 \\
\hline & $b$ & 2.5 & 2.4 & 2.3 & 2.2 & 2.2 \\
\hline & $R^{2}$ & 0.994 & 0.993 & 0.989 & 0.984 & 0.978 \\
\hline
\end{tabular}

processes. One possibility for such processes was conformational changes undergone by the cytochrome $c$ molecules right after adsorption. ${ }^{20)}$ Using the time-resolved SOWG technique that we have developed, more detailed examination of the protein adsorption process can now be observed.

\section{Conclusions}

Our results provided the experimental evidence to support the claim that the first stage of protein adsorption is reversible and irreversibility is initiated with increasing residence time attributed to the secondary and tertiary structural changes that occur as soon as the protein molecules interact with the surface functionalities. Despite the complexity of proteinsurface interactions and a wide disagreement in interpreting the adsorption and desorption of proteins on surfaces, there is, however, a common certainty supported by our results that the first stage of protein adsorption is a reversible process. This reversibility is manifested in the very early stage of protein adsorption characterized by $R^{2}$ values closest to unity when the experimental data are fitted into the simple adsorption model. Although our results did not show any direct evidence of conformational transformation after adsorption, the observed data did not contradict the idea that with prolonged contact, the adsorbed protein molecules undergo some molecular relaxation or spreading on the surface making them more difficult to displace or remove. The SOWG platform we have presented here expediently provided time-resolved absorbance data for probing protein adsorption kinetics and this feature is currently not accessible to conventional total internal reflection-based methods.

\section{REFERENCES}

1) M. Niinomi, T. Hattori, K. Morikawa, T. Kasuga, A. Suzuki, H. Fukui and S. Niwa: Mater. Trans. 43 (2002) 2970-2977.

2) M. Okido, K. Nishikawa, K. Kuroda, R. Ichino, Z. Zhao and O. Takai: Mater. Trans. 43 (2002) 3010-3014.

3) V. Hlady, J. Buijs and H. P. Jennissen: Methods Enzymol. 309 (1999) 402-429.

4) R. E. Dessy: Anal. Chem. 61 (1989) 1079A-1094A.

5) I. Chabay: Anal. Chem. 54 (1982) 1071A-1080A.

6) Z. M. Qi, N. Matsuda, T. Yoshida, H. Asano, A. Takatsu and K. Kato: Optics Letters 27 (2002) 2001-2003.

7) J. H. Santos, N. Matsuda, Z. M. Qi, T. Yoshida, A. Takatsu and K. Kato: Surf. Interface Anal. 35 (2003) 432-436.

8) Z. M. Qi, N. Matsuda, J. Santos, K. Itoh, A. Takatsu and K. Kato: Langmuir 19 (2003) 214-217.

9) J. H. Santos, N. Matsuda, Z. M. Qi, T. Yoshida, A. Takatsu and K. Kato: Anal. Sci. 19 (2003) 199-204.

10) N. Matsuda, J. J. Zheng, D. K. Qing, A. Takatsu and K. Kato: Appl. Spectrosc. 57 (2003) 100-103.

11) J. H. Santos, N. Matsuda, Z. M. Qi, A. Takatsu and K. Kato: IEICE Trans. Electron. E85-C (2002) 1275-1281.

12) N. Matsuda, A. Takatsu, K. Kato and Y. Shigesato: Chem. Lett. (1998) $125-126$.

13) N. Matsuda, A. Takatsu and K. Kato: Chem. Lett. (1996) 105-106.

14) K. Kato, A. Takatsu, N. Matsuda, R. Azumi and M. Matsumoto: Chem. Lett. (1995) 437-438.

15) D. Behrend and K. P. Schmitz: Biomed. Tech. 38 (1993) 172-178.

16) J. R. Bain and A. S. Hoffman: Biomaterials 23 (2002) 3347-3357.

17) D. Hall: Anal. Biochem. 288 (2001) 109-125.

18) P. R. Edwards, A. Gill, D. V. Pollard-Knight, M. Hoare, P. E. Buckle, P. A. Lowe and R. J. Leatherbarrow: Anal. Biochem. 231 (1995) 210217.

19) L. Shi and K. D. Caldwell: J. Colloid Interface Sci. 224 (2000) 372381.

20) X. X. Chen, R. Ferrigno, J. Yang and G. A. Whitesides: Langmuir 18 (2002) 7009-7015 